\title{
Influencios externas en la organización del Ejército colombiono en la primera mitad del siglo xx
}

\section{Resumen}

Lidia Esperanza Alvira Gómez*

La profesionalización y organización del Ejército Nacional fue una tarea emprendida durante el periodo presidencial del general Rafael Reyes. Aunque presentó contradictores, su objetivo fue traer un selecto grupo de militares extranjeros del Ejército chileno. Ellos, con su experiencia y conocimiento, tuvieron a cargo la empresa de estructurar la carrera militar en el siglo xx, organizar la escuela de formación, escribir los manuales y establecer todo lo relacionado con la doctrina militar del país. Dispuesto así, se trajeron cuatro misiones chilenas a partir del año de 1907. Se eligió al Ejército chileno por la trayectoria de su formación militar, iniciada en 1885 cuando este contrató una misión alemana para que lo formara e instruyera en la doctrina militar de importancia a nivel mundial. Desde ese momento y por tal razón, el Ejército chileno se destacó entre los existentes para la época en Latinoamérica. Por este motivo, la profesionalización de la carrera militar en los inicios del siglo xx obedeció a una fuerte influencia chilena -y esta última, a su vez, de la escuela alemana-. Posteriormente, con la participación del Batallón Colombia en la Guerra de Corea, llegó la influencia norteamericana de la mano de la instrucción militar, el armamento, los uniformes y accesorios que recibieron los militares activos colombianos que participaron en la actividad bélica en nombre de los Estados Unidos de Norteamérica.

Palabras clave: Fuerzas Armadas Norteamericanas, misiones, misiones chilenas, profesionalización militar

\section{Abstract}

The professionalization and organization of the National Army was a task undertaken during the presidential term of General Rafael Reyes. Although he presented contradictors, his goal was to bring a select group of foreign military from the Chilean Army. They, with their experience and knowledge, were in charge of structuring the military career in the twentieth century, organizing the training school, writing the manuals and establishing everything related to the military doctrine of the country. Thus, four Chilean missions were brought in from 1907. The Chilean Army was chosen for its military formation, which began in 1885 when it hired a German mission to train and instruct it in the military doctrine of world importance. From that moment, the Chilean Army stood out among those existing for the time in Latin America. For this reason, the professionalization of the military career at the beginning of the twentieth century was due to a strong Chilean influence, and the latter, in turn, to the German school. Later, with the participation of the Colombian Battalion in the Korean War, the US influence came from the hand of military training, the weaponry, uniforms and accessories received by the active Colombian military who participated in the war activity on behalf of the United States of America.

Keywords: Chilean missions, military professionalization, US army

\footnotetext{
* Docente en la Escuela de Comunicación y Bellas Artes de la Corporación Unificada Nacional de Educación Superior (cun). Contacto: lidia_alvira@cun.edu.co
} 


\section{Influencia militar chilena}

Para hablar de la influencia militar chilena en el Ejército colombiano, es importante conocer un poco cómo se preparó Chile antes de llegar al país. Todo surgió "como consecuencia de la Guerra del Pacífico, [coyuntura por la que] el Ejército de Chile vio la necesidad de modernizar y reorganizar sus fuerzas militares" (Arancibia, 2007, p. 180). Es de recordar que Chile fue el primer país en América Latina que tomó la decisión de contratar una misión alemana, hacia 1885, después de analizar la efectividad y las sucesivas victorias demostradas por las filas militares germanas en las tres últimas guerras que habían enfrentado: Dinamarca (1864), Austria (1866) y Francia (1870).

En 1906 se decretó la reorganización general del Ejército bajo el modelo alemán en todos sus aspectos. Este hecho reestructuró el Estado Mayor General del Ejército con miras a cumplir a cabalidad la misión específica de abocarse al estudio y la preparación de los elementos que se podrían requerir en caso de una situación de posible de guerra, como cartas, vías de comunicación, transportes, recursos y todo lo que se refiriera a proyectos de probables planes de operaciones, es decir, el estudio y preparación de las maniobras anuales y la más completa instrucción del personal por medio de viajes, tareas, conferencias y trabajos históricos.

"El tiempo y la dedicación de los adoctrinadores alemanes dejaron su fruto en el ejército chileno [que] ya era un símbolo de respeto, admiración y como consecuencia lo llevaron a convertirse en la primera potencia militar de Sudamérica" (Barros, 2007, p. 164). Por otra parte, factores fundamentales como su ubicación, el idioma, las costumbres similares y el hecho de tener un gobierno austero y progresista fueron las causas que incidieron directamente para que otras naciones de Sur América como Ecuador, Colombia, Venezuela, entre otros, tomaran en cuenta el modelo militar chileno, incluso sobre los oficiales alemanes directamente.

En el caso colombiano, la situación era dramática: en 1902 había culminado una guerra civil entre conservadores y liberales, que sacó triunfante a los primeros, pero que cobró más de 100000 vidas, la destrucción de muchas propiedades y la desmoralización de la vida nacional. A lo anterior, se sumó la pérdida de Panamá en 1903, alcanzada con el apoyo de EE. UU. El general Rafael Reyes, con un pujante proceso revitalizador, se enfrentó a este panorama una vez asumió la presidencia en 1904.

Entre los años 1907 y 1915 hubo cuatro misiones chilenas en Colombia. La primera estuvo conformada por el capitán Arturo Ahumada y el capitán Diego Guillén, que fueron designados como director y subdirector respectivamente de la primera Escuela Militar Colombiana en abril de 1907. Entre sus obras más importantes, se destaca la creación del plan de estudios y la reglamentación de la entidad.

\section{Primera misión chilena}

Bajo la dirección del coronel alemán Emilio Kornel (1843-1920), Chile adquirió los conocimientos de la escuela militar alemana y profesionalizó su ejército durante el periodo comprendido entre 1886 y 1910 (Barros, 2007). Prácticamente, la escuela chilena se convirtió en una academia de guerra con un programa de estudios de tres años y más de treinta oficiales germanos. Entre ellos, se encontraban aquellos que asumirían la labor de remodelar el Ejército de Colombia. En 1907, arrancó la organización 
de nuestro ejército con la formalización de la Escuela Militar de Cadetes, acto sancionado por el Decreto 434 del 13 abril de 1907.

La misión chilena tenía dentro de sus obligaciones contractuales renovar el alto mando del Ejército colombiano, elaborar un reglamento orgánico y crear la disposición operativa de las fuerzas que se agruparon. Era crucial cumplir los acuerdos establecidos entre el Gobierno colombiano y la misión chilena durante la vigencia del contrato celebrado, que fue firmado por el comandante de la Escuela, capitán Arturo Ahumada Bascuña, y su segundo, el capitán Diego Guillén Santana. Dicho contrato, amparado por el voto de confianza y satisfacción del Ministerio, obligaba a los capitanes a servir como oficiales durante cuatro años, so pena de pagar \$100 por cada año de incumplimiento. Ellos recibieron sus sueldos, derechos y preeminencias de su grado, más un sobresueldo de \$13 por concepto de alimentación y casino, y \$6 para cubrir gastos de libros. Además, el Gobierno decretó la formación de una batería de artillería y de un batallón de infantería modelos para que la tropa recibiera, en el marco de estas unidades, la instrucción de la misión chilena. Inicialmente, la Escuela funcionó con dos cursos, a saber, el curso militar para oficiales alumnos y el curso para cadetes, denominado Curso general. La duración de los cursos se estableció en cuatro años, aunque en el camino se realizaron modificaciones que permitieron lograr los objetivos que se buscaban. Esta misión también sustituyó el antiguo sistema de rancho de la tropa por el empleado en Chile. Este se mantiene hasta el día de hoy. El sistema para los soldados consistía en comer en el suelo con la compañera indígena y se les mantenía en las filas por medio del azote.

Desde ese momento, el fisco empezó a pagar la comida del soldado de tropa, lo que dio fin a la costumbre de que cada uno costeara su alimento. Esta nueva política finalmente evitó que los soldados llevaran al cuartel mujeres para que les acompañasen y cocinaran, hechos que, entre otras cosas, en ocasiones provocaban desorden, promiscuidad y aumento del consumo de bebidas alcohólicas basadas en granos. Para 1909, la misión chilena sospechaba que Reyes estaba usando la profesionalización militar para sus beneficios personales. Como consecuencia de la confirmación de sus sospechas, la misión chilena se enfureció y pronunció su renuncia.

\section{Segunda misión chilena}

Después de fracasar en su intento de normalizar las relaciones con Estados Unidos, Reyes abandonó el poder en 1909. Tras su renuncia, el general Jorge Holguín asumió la Presidencia de la República. Recibió el poder el 9 de junio de 1909 y lo entregó el 4 de agosto de ese año al cederle el poder de presidente titular al general Ramón González Valencia hasta el 7 de agosto de 1910. La segunda misión militar chilena inició bajo este contexto a comienzos de 1910. Estuvo abanderada por los capitanes Francisco Javier Díaz y Pedro Charpin. Llegaron a Colombia en un clima de alta tensión política suscitada por las elecciones y la firma del tratado que aceptó la independencia de Panamá. Pero, de inmediato, su presencia causó descontento en algunos mandatos militares debido a su pretensión de nombrar liberales en algunos ministerios. Acto seguido a su designación, el ministro de Guerra convocó a los comandantes de las fuerzas formadas para forjar el golpe. El capitán Díaz asumió la dirección de la Escuela Militar y, por su parte, el capitán Charpin fue nombrado primer director de la recién formada Escuela Superior de Guerra. La principal tarea de esta misión fue la organización de esta Escuela, que consideró inicialmente un curso de Estado mayor, de un año de duración, y un curso de aplicación, de seis meses. La misión chilena, tras enterarse de los preparativos 
para el golpe, encabezada por Francisco Javier Díaz y Pedro Charpin Rival, manifestó su renuncia verbal ante notaría, ministro y en las páginas de la Gaceta Republicana (periódico de la época):

Como las leyes (del código de honor) califican como delito de alta traición lo que pretende del Ejercito; como en nuestro carácter de extranjeros no podemos protestar ante el atentado que se prepara y como en nuestros carácter de militares menos podemos dar, como nuestra pasividad o nuestro silencio, semejante lección a los jóvenes que estamos educando en la carrera de la lealtad y del deber, nos vemos obligados a presentar ante SS renuncia irrevocable de los puestos que nos han sido confiados, para dirigirnos inmediatamente a nuestro país. (Atehortúa y Vélez, 1994, p. 67)

\section{La tercera misión}

Estuvo compuesta por tres oficiales, pese a que el Gobierno colombiano había solicitado cinco: el mayor de infantería Washington Montero Carvallo, el capitán de artillería Pedro Vignola y el capitán de ingenieros Manuel Aguirre. A estos oficiales se les confirió el grado de coronel, al primero, y de mayor, a los dos capitales. Llegan a Bogotá en marzo de 1912. El mayor Montero asumió la dirección de la Escuela Militar, entre otra serie de responsabilidades, el capitán Vignola se desempeñó como instructor de armas montadas y profesor en la Escuela Superior de Guerra, y el capitán Aguirre fungió como instructor de ingenieros y profesor en ambas escuelas (Aranciba, 2007).

\section{La cuarta misión}

Inició en junio de 1913. Estuvo a cargo del mayor Carlos Sáez Morales, que se dedicó a preparar leyes militares sobre requisitos de ingreso al Ejército y sobre aspectos de material de guerra. Sáez también se desempeñó como director de la Escuela Militar y como profesor en la Escuela Superior de Guerra.

Terminó su labor tras presentar su renuncia forzada en 1915 por causa de intromisiones políticas del Gobierno. En conclusión, se puede afirmar que el Ejército colombiano fue formado, organizado y adoctrinado por el Ejército chileno, dentro de cuyas virtudes se destaca su estricta disciplina y nivel de exigencia. Por otro lado, cabe subrayar que los uniformes fueron otro aporte significativo del Ejército chileno. Este último, a su vez, recibió esta influencia de las fuerzas militares alemanas, como queda demostrado a través de las siguientes fotografías:

Figura 1. Soldados alemanes principios del siglo xx (registro fotográfico del Museo Militar, Sala Ejército Nacional de Colombia, Bogotá, 2014)

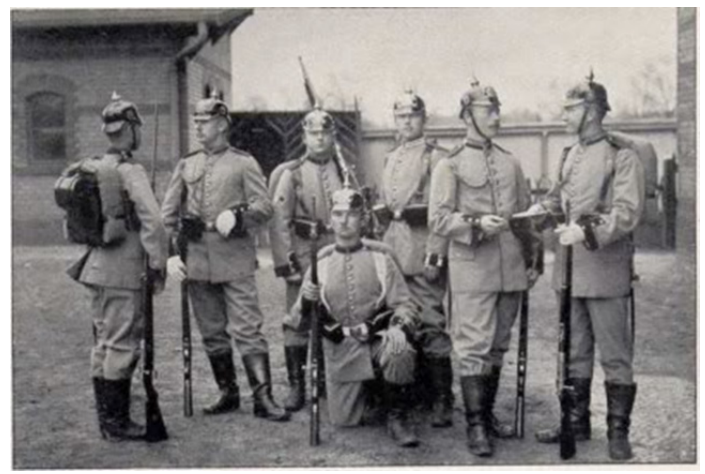

Fuente: registro fotográfico del Museo Militar, Sala Ejército Nacional de Colombia, Bogotá, 2014. 
Figura 2. Militares chilenos en 1902

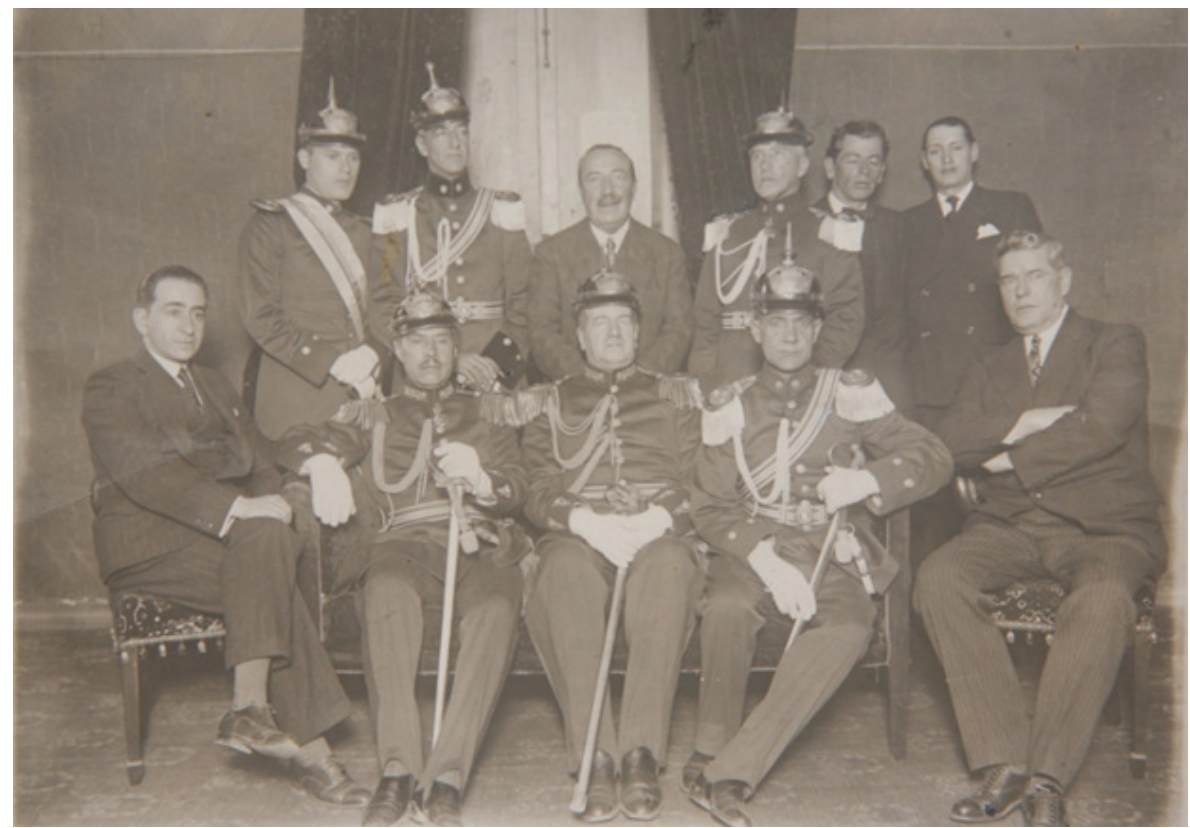

Fuente: registro fotográfico del Museo Militar, Sala Ejército Nacional de Colombia, Bogotá, 2014.

Figura 3. Formación Escuela Militar Bogotá-San Diego

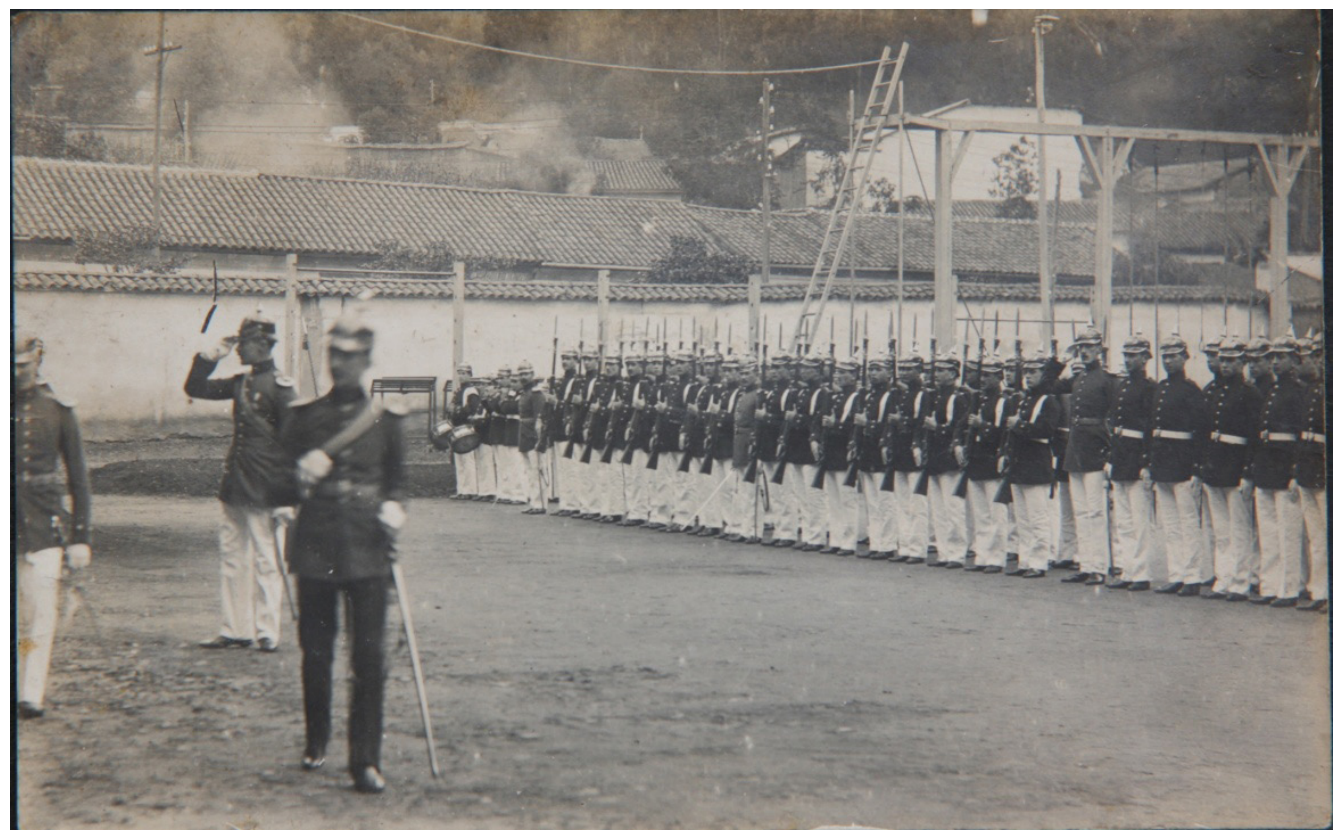

Fuente: registro fotográfico del Museo Militar, Sala Ejército Nacional de Colombia, Bogotá, 2014. 


\section{Influencia militar norteamericana}

De acuerdo con algunos testimonios de veteranos de la Guerra de Corea, Colombia recibió instrucción militar de los cuadros norteamericanos que vinieron al país a preparar específicamente al grupo seleccionado del Ejército para conformar el Batallón Colombia. A partir de ese momento, los funcionarios adquieren sus conocimientos, doctrina, uniformes y armamento. Después de ese lapso, los jóvenes militares colombianos tuvieron contacto con un país más avanzado tecnológicamente y con recursos que invierte en la adquisición de uniformes, accesorios y armamento. Según el testimonio del veterano de la Guerra de Corea Isaac Vargas Córdoba, "un fusil M1 era el [que] se usaba para finales de los años cuarenta, que la exigencia en el aseo era muy alta [sic]" (Vargas, 12 de septiembre de 2013). Los grupos de militares colombianos que participaron en la Guerra de Corea recibieron uniformes y armamento americano de color verde, mientras que, en el país, el uniforme es de color caqui.

De acuerdo con el testimonio de Carlos Alberto Pantorra Franco, para 1953 los soldados usaban "un fusil máuser de cerrojo" y "el Batallón Colombia era autónomo, porque nosotros no recibíamos órdenes directas de los gringos, únicamente el comando del batallón recibía las ordenes y el comando del batallón repartía al batallón Colombia, pero nosotros directamente no teníamos contacto" (Pantorra, 19 de septiembre del 2013). El testimonio del cabo segundo Pantorra sugiere una diferencia, a saber, que el armamento que tenían para el año 1953 en el país, al parecer, es diferente al M1 que menciona el sargento Vargas. Con relación a las órdenes, el Batallón Colombia está agrupado y es el comando el que las recibe directamente de los gringos, como se les dice coloquialmente a los norteamericanos. Por su parte, si bien los militares de bajo rango no tuvieron trato directo con el Ejército norteamericano, sí recibieron uniformes y armamento de parte de él, así como instrucción militar en Colombia. Al respecto, el sargento segundo José Vidal Beltrán Molano relata lo siguiente:

Teníamos que someternos a un entrenamiento aquí en Colombia de noventa días en la escuela de infantería. Yo trabajaba en esa época ya como cabo primero en el Guardia Presidencial [sic]. Teníamos que someternos a un entrenamiento muy fuerte en la Escuela de Infantería, dirigida por algunos oficiales americanos, y luego nos practicaron un examen físico, y luego salíamos para Corea. (28 de septiembre de 2013)

Como se sabe, el Gobierno colombiano pertenecía al grupo de los aliados; por eso envía voluntarios para apoyar en la Guerra de Corea. En contraprestación por la ayuda, Colombia recibió instrucción militar dentro del país dirigida por personal militar americano, en especial, para entrenar al grupo de militares que participaría en Corea. 


\section{Referencias}

Arancibia, R. (2007). La influencia del Ejército de Chile en América Latina 1900-1960. En Academia de Historia Militar (ed.), Anuario de la Academia de Historia Militar de Chile (Edición especial 30 años) (pp. 180-203). Santiago de Chile: Academia de Historia Militar.

Atehortúa, A. y Vélez, H. (1994). Estado y Fuerzas Armadas en Colombia: 1886-1953. Bogotá: Tercer Mundo Editores; Universidad Javeriana (Cali).

Barros, M. (2007). El general Emilio Körner Henze en la reorganización del Ejército de Chile 1885-1910. En Academia de Historia Militar (ed.), Anuario de la Academia de Historia Militar de Chile (Edición especial 30 años) (pp. 161-179). Santiago de Chile: Academia de Historia Militar.

Museo Militar de Colombia. (s. f.). Formación Escuela Militar Bogotá-San Diego [Fotografía]. Bogotá, Museo Militar de Colombia, Sala Ejército Nacional de Colombia.

Museo Militar de Colombia. (s.f.). Militares chilenos en 1902 [Fotografía]. Bogotá, Museo Militar de Colombia, Sala Ejército Nacional de Colombia.

Museo Militar de Colombia. (s.f.). Soldados alemanes principios del siglo xx [Fotografía]. Bogotá, Museo Militar de Colombia, Sala Ejército Nacional de Colombia.

Pantorra, C. (19 de septiembre del 2013). Entrevista con el cabo segundo Carlos Alberto Pantorra Franco [comunicación personal].

Presidencia de la República de Colombia. (13 de abril de 1907). Decreto 434 de 1907: por el cual se organiza la Escuela Militar.

Vargas, I. (12 de septiembre del 2013). Entrevista con el sargento primero Isaac Vargas Córdoba [comunicación personal].

Vidal, J. (28 de septiembre del 2013). Entrevista con el sargento segundo José Molano Vidal Beltrán [comunicación personal]. 